\title{
BMC Cardiovascular Disorders reviewer acknowledgement 2015
}

Tim Shipley

\section{Contributing reviewers}

The editors of BMC Cardiovascular Disorders would like to thank all our reviewers who have contributed to the journal in Volume 15 (2015).

Flavia Ballocca
Italy

Matteo Bianco

Italy

Antonio H Frangieh

Switzerland

Franck Boccara

France

Sebastiano Gili

Italy

Karoly Kaszala

USA

Guido A. Matschuck Mhba

Germany

Robin Dullaart

Netherlands

John Hughes

UK

Toshinori Kamisako

Japan

Ryan Lennon

USA

Eiji Oda

Japan
Tony Merriman

New Zealand

Barry Palmer

New Zealand

Liang Chen

China

Andrea Chomistek

USA

Hannu Vanhanen

Finland

Umberto Benedetto

UK

Antonio Miceli

Italy

Susumu Manabe

Japan

Angelos Rigopoulos

Germany

Simone Biscaglia

Italy

Mario Iannaccone

Italy

Veronica D' Annunzio

Argentina
Liliana Nicolosi

Argentina

Dionyssios Leftheriotis

Greece

Vasilis Stasinos

Greece

Aldo Clerico

Italy

Armando Pucciarelli

Italy

Davide Giacomo Presutti

Italy

Giorgio Quadri

Italy

Fabrizio D'Ascenzo

Italy

Sven Meyer

Germany

Martina Zaninotto

Italy

Wayne Smith

South Africa

Julia Braun

Switzerland

Correspondence: tim.shipley@biomedcentral.com

BioMed Central, Floor 6, 236 Gray's Inn Road, London WC1X 8HB, UK 


\begin{tabular}{|c|c|c|}
\hline $\begin{array}{l}\text { Geetha Chittoor } \\
\text { USA }\end{array}$ & $\begin{array}{l}\text { Ibrahim Akin } \\
\text { Germany }\end{array}$ & $\begin{array}{l}\text { Ammar Ashor } \\
\text { UK }\end{array}$ \\
\hline $\begin{array}{l}\text { Francesca Viazzi } \\
\text { Italy }\end{array}$ & $\begin{array}{l}\text { Michael Behnes } \\
\text { Germany }\end{array}$ & $\begin{array}{l}\text { Henner Hanssen } \\
\text { Switzerland }\end{array}$ \\
\hline $\begin{array}{l}\text { Hippokratis Kiaris } \\
\text { Greece }\end{array}$ & $\begin{array}{l}\text { Stefano Pidello } \\
\text { Italy }\end{array}$ & $\begin{array}{l}\text { Hsin-Fu Lin } \\
\text { Taiwan }\end{array}$ \\
\hline $\begin{array}{l}\text { Jian Xiao } \\
\text { China }\end{array}$ & $\begin{array}{l}\text { Marta Bisi } \\
\text { Italy }\end{array}$ & $\begin{array}{l}\text { Juan Gagliardi } \\
\text { Argentina }\end{array}$ \\
\hline $\begin{array}{l}\text { Brian Cooley } \\
\text { USA }\end{array}$ & $\begin{array}{l}\text { Marco Pavani } \\
\text { Italy }\end{array}$ & $\begin{array}{l}\text { Manuel Rodriguez } \\
\text { Argentina }\end{array}$ \\
\hline $\begin{array}{l}\text { Salma Esmaeil } \\
\text { Egypt }\end{array}$ & $\begin{array}{l}\text { Anna Grodzinsky } \\
\text { USA }\end{array}$ & $\begin{array}{l}\text { Alexander Berndt } \\
\text { Germany }\end{array}$ \\
\hline $\begin{array}{l}\text { Rumi Faizer } \\
\text { USA }\end{array}$ & $\begin{array}{l}\text { Dany Jacob } \\
\text { USA }\end{array}$ & $\begin{array}{l}\text { Katja Grün } \\
\text { Germany }\end{array}$ \\
\hline $\begin{array}{l}\text { Eduardo Saadi } \\
\text { Brazil }\end{array}$ & $\begin{array}{l}\text { Carmelina Ariano } \\
\text { Italy }\end{array}$ & $\begin{array}{l}\text { Isaac Aidonidis } \\
\text { Greece }\end{array}$ \\
\hline $\begin{array}{l}\text { Christian Delles } \\
\text { UK }\end{array}$ & $\begin{array}{l}\text { Imke Christiaans } \\
\text { Netherlands }\end{array}$ & $\begin{array}{l}\text { Panagiota Flevari } \\
\text { Greece }\end{array}$ \\
\hline $\begin{array}{l}\text { Julian Walters } \\
\text { UK }\end{array}$ & $\begin{array}{l}\text { Clemens Von Schacky } \\
\text { Germany }\end{array}$ & $\begin{array}{l}\text { Evangelos Repasos } \\
\text { Greece }\end{array}$ \\
\hline $\begin{array}{l}\text { Ignatios Ikonomidis } \\
\text { Greece }\end{array}$ & $\begin{array}{l}\text { Jun Hu } \\
\text { USA }\end{array}$ & $\begin{array}{l}\text { Marijana Tadic } \\
\text { Serbia }\end{array}$ \\
\hline $\begin{array}{l}\text { Agathi-Rosa Vrettou } \\
\text { Greece }\end{array}$ & $\begin{array}{l}\text { Linlin Li } \\
\text { USA }\end{array}$ & $\begin{array}{l}\text { Gianluca Campo } \\
\text { Italy }\end{array}$ \\
\hline $\begin{array}{l}\text { Antonio Loforte } \\
\text { Italy }\end{array}$ & $\begin{array}{l}\text { Guangliang Wang } \\
\text { USA }\end{array}$ & $\begin{array}{l}\text { Yoichi Imori } \\
\text { Switzerland }\end{array}$ \\
\hline $\begin{array}{l}\text { Maria Luisa Granada } \\
\text { Spain }\end{array}$ & $\begin{array}{l}\text { Abdulrahman Al-Malki } \\
\text { Saudi Arabia }\end{array}$ & $\begin{array}{l}\text { Johannes Kragten } \\
\text { Netherlands }\end{array}$ \\
\hline $\begin{array}{l}\text { Igor Koncar } \\
\text { Serbia }\end{array}$ & $\begin{array}{l}\text { Daniel Conklin } \\
\text { USA }\end{array}$ & $\begin{array}{l}\text { Asru Kumar Sinha } \\
\text { India }\end{array}$ \\
\hline $\begin{array}{l}\text { Emiliano Angeloni } \\
\text { Italy }\end{array}$ & $\begin{array}{l}\text { Siyavash Joukar } \\
\text { Iran }\end{array}$ & $\begin{array}{l}\text { Sanjiv Dhingra } \\
\text { Canada }\end{array}$ \\
\hline $\begin{array}{l}\text { Ana Olga Mocumbi } \\
\text { Mozambique }\end{array}$ & $\begin{array}{l}\text { Chiara Davico } \\
\text { Italy }\end{array}$ & $\begin{array}{l}\text { Ian Mcniece } \\
\text { USA }\end{array}$ \\
\hline $\begin{array}{l}\text { A Sampath Kumar } \\
\text { India }\end{array}$ & $\begin{array}{l}\text { Marianna Mazza } \\
\text { Italy }\end{array}$ & $\begin{array}{l}\text { Tiina Heliö } \\
\text { Finland }\end{array}$ \\
\hline $\begin{array}{l}\text { Guillermo Isasti } \\
\text { Spain }\end{array}$ & $\begin{array}{l}\text { Alvaro Alonso } \\
\text { USA }\end{array}$ & $\begin{array}{l}\text { Jelena Seferovic } \\
\text { Serbia }\end{array}$ \\
\hline $\begin{array}{l}\text { Raul Lopez-Salguero } \\
\text { Spain }\end{array}$ & $\begin{array}{l}\text { Weihong Tang } \\
\text { USA }\end{array}$ & $\begin{array}{l}\text { Zehra Bugra } \\
\text { Turkey }\end{array}$ \\
\hline $\begin{array}{l}\text { María José Molina Mora } \\
\text { Spain }\end{array}$ & $\begin{array}{l}\text { Jackie Boucher } \\
\text { USA }\end{array}$ & $\begin{array}{l}\text { Vincent Robinson } \\
\text { USA }\end{array}$ \\
\hline
\end{tabular}


David Carty

UK

Carmel Mceniery

UK

David Freedman

USA

William Johnson

UK

Markus Juonala

Finland

Dario Celentani

Italy

Ping-Yen Liu

Taiwan

Vikram Agarwal

USA

Chirag Bavishi

USA

Parasuram Krishnamoorthy

USA

Kimberley Mellor

New Zealand

Michele Malaguarnera

Italy

Olga Mcleod

Sweden

Francesca Giordana

Italy

Gilles Lemesle

France

Marcus Franz

Germany

Thomas Meyer

Germany

Jeetesh Patel

UK

Peter Rahko

USA

Alistair Stewart

New Zealand

Madhulika Dixit

India

Yehuda Kamari

Israel
Jeffrey Brinker

USA

Ramez Nairooz

USA

Rita Pavasini

Italy

Michinari Nakamura

USA

Arintaya Phrommintikul

Thailand

Wilbert Aronow

USA

Bredy Pierre-Louis

USA

Devender Dhanda

USA

Giuseppina Di Biase

Italy

Marco Mennuni

Italy

Giulia Nangeroni

Italy

Lizhang Chen

China

Jingpu Shi

China

George Andrikopoulos

Greece

Dimitris Tsiachris

Greece

Fausto Biancari

Finland

Massimo Imazio

Italy

Na Li

USA

Michael Lipinski

USA

Michael Schwarzer

Germany

Lei Teng

USA

Yixuan Zhang

USA
Kurt Bestehorn

Germany

Michael Zairis

Greece

Barbro Kjellström

Sweden

Corey Tomczak

Canada

Amir Gahremanpour USA

Yunn-Hwa Ma

Taiwan

Balázs Magyari

Hungary

Martina Pianelli

Italy

M.-Saadeh Suleiman

UK

Daniela Mazzaccaro

Italy

Giovanni Nano

Italy

Harry Smolen

USA

Shirling Lin

Taiwan

Jose Mill

Brazil

Charles Mondo

Uganda

Ayotunde Dokun

USA

Andrew Klein

USA

Naoyuki Takashima

Japan

Marek Brabec

Republic Czech

Christian Heiss

Germany

Antonis Vlassopoulos

Switzerland

Josef Dankiewicz

Sweden 
Tinne Tranberg

Denmark

Sebastian Wiberg

Denmark

Oliver Gunkel

Germany

Mingyi Wang

USA

Vicky Cameron

New Zealand

Christopher Wong

UK

Spyridon Katsanos

Greece

Stamatios Lerakis

USA

Stylianos Orfanos

Greece

Nobuyuki Ohte

Japan

Danica Chen

USA

Augusto Montezano

UK

Ravi Sundaresan Nagalingam

India

Kai Lin

USA

Hajime Sakuma

Japan

Kyungeh An

USA

Wenqi Gan

USA

Peter Willeit

UK

Alessio Alogna

Austria

Heiner Post

Germany

Bronwyn Brown

Australia

Caroline Genco

USA
Mirela Habibovic

Netherlands

Kim Smolderen

USA

Kim Smolderen

Netherlands

Michael Stickland

Canada

Massimo Chello

Italy

Mario Petretta

Italy

Laura Perin

USA

Rajesh Ramasamy

Malaysia

Ntobeko Ntusi

South Africa

Welma Stonehouse

Australia

Welma Stonehouse

New Zealand

Jason Gill

UK

Raj Kumar Yadav

India

Branislava Ivanovic

Serbia

Sorina Mihaila

Romania

Grzegorz Bilo

Italy

Malgorzata Kloch-Badelek

Poland

Yu Jin

Belgium

Wiktoria Wojciechowska

Poland

Bo $\mathrm{Hu}$

USA

Robert Biederman

USA

Saurabh Gupta

India
Ignacio Lugones

Argentina

Alanna Chamberlain

USA

Massimo Zoni-Berisso

Italy

Silvia Punzetti

Italy

Stefano Volpato

Italy

Thomas Gremmel

Austria

Konstantinos Katsanos

Greece

Paola Rizzo

Italy

Krisztina Heltai

Hungary

Lakshmi Mundkur

India

Emilio Vanoli

Italy

Tze-Fan Chao

Taiwan

Shao-Yuan Chuang

Taiwan

Chao-Yu Guo

Taiwan

Thomas Rosemann

Switzerland

Guillaume Lettre

Switzerland

Sebastien Theriault

Canada

Vasiliki Betihavas

Australia

David Schopfer

USA

Giacomo Boccuzzi

Italy

Alice Owen

Australia

David Preiss

UK 


\begin{tabular}{|c|c|c|}
\hline $\begin{array}{l}\text { Li Jia } \\
\text { China }\end{array}$ & $\begin{array}{l}\text { Tadeusz Osadnik } \\
\text { Poland }\end{array}$ & $\begin{array}{l}\text { Julia Grapsa } \\
\text { UK }\end{array}$ \\
\hline $\begin{array}{l}\text { Yu Lu } \\
\text { USA }\end{array}$ & $\begin{array}{l}\text { Teresa Villarreal-Molina } \\
\text { Mexico }\end{array}$ & $\begin{array}{l}\text { Kamal Mubarak } \\
\text { USA }\end{array}$ \\
\hline $\begin{array}{l}\text { Amr Gad } \\
\text { Egypt }\end{array}$ & $\begin{array}{l}\text { Ju Yi Chen } \\
\text { Taiwan }\end{array}$ & $\begin{array}{l}\text { Victoria Delgado } \\
\text { Netherlands }\end{array}$ \\
\hline $\begin{array}{l}\text { Giovanni Melina } \\
\text { Italy }\end{array}$ & $\begin{array}{l}\text { Pai Feng Hsu } \\
\text { Taiwan }\end{array}$ & $\begin{array}{l}\text { Knut Matre } \\
\text { Norway }\end{array}$ \\
\hline $\begin{array}{l}\text { Xiangwei Wu } \\
\text { China }\end{array}$ & $\begin{array}{l}\text { Martin Schultz } \\
\text { Australia }\end{array}$ & $\begin{array}{l}\text { Marjukka Kolehmainen } \\
\text { Finland }\end{array}$ \\
\hline $\begin{array}{l}\text { Surendra Chutani } \\
\text { India }\end{array}$ & $\begin{array}{l}\text { Lin Xu } \\
\text { Hong Kong }\end{array}$ & $\begin{array}{l}\text { Giuseppe Ferrante } \\
\text { Italy }\end{array}$ \\
\hline $\begin{array}{l}\text { Decebal Gabriel Latcu } \\
\text { Monaco }\end{array}$ & $\begin{array}{l}\text { Daniel Petrovic } \\
\text { Slovenia }\end{array}$ & $\begin{array}{l}\text { Gennaro Giustino } \\
\text { USA }\end{array}$ \\
\hline $\begin{array}{l}\text { Hassan Behlouli } \\
\text { Canada }\end{array}$ & $\begin{array}{l}\text { Naoki Sato } \\
\text { Japan }\end{array}$ & $\begin{array}{l}\text { Masahiro Natsuaki } \\
\text { Japan }\end{array}$ \\
\hline $\begin{array}{l}\text { Amresh Raina } \\
\text { USA }\end{array}$ & $\begin{array}{l}\text { Dmitry Shchekochikhin } \\
\text { Russian Federation }\end{array}$ & $\begin{array}{l}\text { Giuseppe Merla } \\
\text { Italy }\end{array}$ \\
\hline $\begin{array}{l}\text { Dick Chan } \\
\text { Australia }\end{array}$ & $\begin{array}{l}\text { Torsten Hansen } \\
\text { Germany }\end{array}$ & $\begin{array}{l}\text { David Buys } \\
\text { USA }\end{array}$ \\
\hline $\begin{array}{l}\text { Thomas Pulinilkunnil } \\
\text { Canada }\end{array}$ & $\begin{array}{l}\text { Arti Shinde } \\
\text { USA }\end{array}$ & $\begin{array}{l}\text { Mizanur Rahman } \\
\text { Bangladesh }\end{array}$ \\
\hline $\begin{array}{l}\text { Mahdi Najafi } \\
\text { Iran }\end{array}$ & $\begin{array}{l}\text { Xiaofang Lu } \\
\text { USA }\end{array}$ & $\begin{array}{l}\text { Bardia Arabkhani } \\
\text { Netherlands }\end{array}$ \\
\hline $\begin{array}{l}\text { Japhet Olisekodiaka } \\
\text { Nigeria }\end{array}$ & $\begin{array}{l}\text { Matthias Pauschinger } \\
\text { Germany }\end{array}$ & $\begin{array}{l}\text { Denise Baricocchi } \\
\text { Italy }\end{array}$ \\
\hline $\begin{array}{l}\text { Hasan Shohag } \\
\text { Australia }\end{array}$ & $\begin{array}{l}\text { Luca Fileti } \\
\text { Italy }\end{array}$ & $\begin{array}{l}\text { Stjepan Jurisic } \\
\text { Germany }\end{array}$ \\
\hline $\begin{array}{l}\text { Christoph Sinning } \\
\text { Germany }\end{array}$ & $\begin{array}{l}\text { Arindam Maitra } \\
\text { India }\end{array}$ & $\begin{array}{l}\text { Emanuela Bostjancic } \\
\text { Slovenia }\end{array}$ \\
\hline $\begin{array}{l}\text { Miyuki Tsuchihashi-Makaya } \\
\text { Japan }\end{array}$ & $\begin{array}{l}\text { Anna Lipowicz } \\
\text { Poland }\end{array}$ & $\begin{array}{l}\text { Yasuhide Kuwabara } \\
\text { Japan }\end{array}$ \\
\hline $\begin{array}{l}\text { Amber Courville } \\
\text { USA }\end{array}$ & $\begin{array}{l}\text { Mads Nybo } \\
\text { Denmark }\end{array}$ & $\begin{array}{l}\text { Nina Zidar } \\
\text { Slovenia }\end{array}$ \\
\hline $\begin{array}{l}\text { Masakazu Hirata } \\
\text { Japan }\end{array}$ & $\begin{array}{l}\text { Francesco Perticone } \\
\text { Italy }\end{array}$ & $\begin{array}{l}\text { Tobias Dünnwald } \\
\text { Austria }\end{array}$ \\
\hline $\begin{array}{l}\text { Bernhard Maisch } \\
\text { Germany }\end{array}$ & $\begin{array}{l}\text { Xin Tu } \\
\text { China }\end{array}$ & $\begin{array}{l}\text { Yogish Kudva } \\
\text { USA }\end{array}$ \\
\hline $\begin{array}{l}\text { Arsen Ristic } \\
\text { Serbia }\end{array}$ & $\begin{array}{l}\text { Robert Klautz } \\
\text { Netherlands }\end{array}$ & $\begin{array}{l}\text { Erwan Leclair } \\
\text { Canada }\end{array}$ \\
\hline $\begin{array}{l}\text { Gurumurthy Hiremath } \\
\text { USA }\end{array}$ & $\begin{array}{l}\text { Yuqian Bao } \\
\text { China }\end{array}$ & $\begin{array}{l}\text { Anup Karan } \\
\text { India }\end{array}$ \\
\hline $\begin{array}{l}\text { Gen Suzuki } \\
\text { USA }\end{array}$ & $\begin{array}{l}\text { Takeshi Matsumura } \\
\text { Japan }\end{array}$ & $\begin{array}{l}\text { Luca Mascitelli } \\
\text { Italy }\end{array}$ \\
\hline
\end{tabular}


Tim Palmer

UK

John Hart

USA

Erik Holy

Switzerland

Patrick Mark

UK

Marius Miglinas

Lithuania

Naveed Akbar

UK

Louise Naylor

Australia

Pupalan Iyngkaran

Australia

Eric Loucks

USA

Paul Fedak

Canada

Katsuhito Fujiu

Japan

Frederic Jacques

Canada

Jiang Huai Wang

Ireland

Alida Caforio

Italy

Jitka Seidlerová

Czech Republic

Matteo Tebaldi

Italy

Jan Krejci

Czech Republic

Kelley Branch

USA

Muhib Khan

USA

Evan Thacker

USA

Abdallah Al-Mohammad

UK

Claudio Borghi

Italy
Giuseppe Giugliano

Italy

Regis Lamberts

New Zealand

Rainer G.H. Moosdorf

Germany

Natasa Bogavac-Stanojevic

Serbia

Nadia Hamdy

Egypt

Kim Connelly

Canada

David Playford

Australia

Jesse Dawson

UK

Moutaz Elkadri

UK

Charles Pearman

UK

Kerstin Hogg

Canada

Stephan Schirmer

Germany

Niels Van Royen

Netherlands

Manabu Hayakawa

Japan

Matteo Ghione

Italy

Fabio Mangiacapra

Italy

Michael Czubryt

Canada

Francesco Paneni

Sweden

Laura Burgers

Netherlands

Simone Huygens

Netherlands

Jian Xiong Chen

USA

Prasad Katakam

USA
Bin Wei

USA

Donal O'Gorman

Ireland

Georgios Theodorakis

Greece

Takumi Yamada

USA

Camille Lassale

UK

Yu-Guo Chen

China

Massimo Iacoviello

Italy

Marinella Tricarico

Italy

Sungjin Chung

South Korea

Tadashi Yoshida

Japan

Xiangjun Zeng

China

Wei Chen

China

Jennifer Strande

USA

Kirstine Calloe

Denmark

Douglas Curran-Everett

USA

Antony Jackson

UK

Morten Olesen

Denmark

Ander Regueiro Cueva

Canada

Majid Ghayour

Iran

Cyril Mamotte

Australia

Camilla Calvieri

Italy

Alfredo Rodriguez

Argentina 
Amelia Carro

Spain

Martin Donato

Argentina

Federico Conrotto

Italy

Rami Doukky

USA

Venkatesh Murthy

USA

Randall Thompson

USA

Rozenn Quarck

Belgium

Mark Toshner

UK

Yoshihiro Fukumoto

Japan

Ana Savic-Radojevic

Serbia

Lei Wei

USA

Luigi Barberini

Italy

Trayambak Basak

USA

Sina Andalib

Islamic Republic Of Iran

Ricardo Perez De La Hoz

Argentina

Mehrdad Larry

Islamic Republic Of Iran

Aurelian Bidulescu

USA

Sayan Sen

UK

Susanne Unverzagt

Germany

Corstiaan Uil

Netherlands

Michael Bursztyn

Israel

Megan Wenner

USA
Mackram Eleid

USA

Oluseun O. Alli

USA

Hongyu Wang

China

Ichiro Tatsuno

Japan

Florian Rader

USA

Frangiscos Parthenakis

Greece

Angel Sanchez-Recalde

Spain

Salah Said

Netherlands

Jamilah Al Rahimi

Saudi Arabia

Ali Shafiq

USA

Matthew Jankowich

USA

S Andreas

Germany

Imran Iftikhar

USA

Pablo Salinas

Spain

Sang Hong Baek

Democratic People'S Republic Of

Korea

Shozo Sueda

Japan

Stavros Tzortzis

Greece

Alexandra Frogoudaki

Greece

Jeffrey A. Brinker

USA

Masanori Taniwaki

Japan

Alessio Mattesini

Italy
Bledar Daka

Sweden

Sukhjinder Nijjer

UK

David Vivas

Spain

Jan Vojacek

Czech Republic

Robert Bauer

USA

Marijana Tadic Serbia And

Montenegro

Marcio Kiuchi

Brazil

Takeshi Takami

Japan

Neal Benowitz

USA

Jamie Hartmann-Boyce

UK

Agathi Vrettou

Greece

Spiridon Katsanos

Greece

Gianluca Faggioli

Italy

P De Rango

Italy

Muhib Khan

USA

Zhu-Ming Zhang

USA

Yuichiro Yano

USA

Inmaculada Moreno

USA

José Manuel García Pinilla

USA

Noela Rodríguez-Losada USA

Alberto Crottogini

Argentina 
Jai Singh

UK

\section{Szu-Yuan Li}

Taiwan, Republic Of China

\section{Szu-Chia Chen}

Taiwan, Republic Of China

Thanh Phan

Australia

\section{Alfonso Ielasi}

Italy

Stella Stabouli

Greece

Sanaz Sedaghat

Netherlands

Ahmet Karabulut

Turkey

Salvatore Carbone

Italy

Rebecca Levitt

USA

Kelvin Wong

Australia

Yunlong Huo

China

Ellen Freel

UK

Tatiana Kuznetsova

Belgium

Melinda Carrington

Australia

Adelaide Fusco

Italy

Masao Saotome

Japan

Eric Lopatta

Germany

James Moon

UK

João Cavalcante

USA

Luca Di Lullo

Italy

Seiji Umemoto

Japan
Ryan Lennon

USA

Douglas Curran-Everett

USA

Sebastian Garcia Zamora

Argentina

Alexander Fuernkranz

Germany

Zachary Bloomgarden

USA

Zdenek Turek

Czech Republic

Hynek Riha

Czech Republic

Salvatore Di Stefano

Spain

Emiliano Rodríguez-Caulo

Spain

Suzanne Van Dijk

Netherlands

Tatjana Rundek

USA

Warren Gasper

USA

Mark Koelemay

Netherlands

Sammy Chan

Canada

Prem Shekar

USA

Carlos Porras

Spain

Kim Smolderen

USA

Agnieszka Kapłon-Cieślicka

Poland

Una Martin

UK

Cornelia Deutsch

Germany

Sreek Vemulapalli

USA

Massimo Fineschi

Italy
Wayne L. Miller

USA

George Theodorakis

Greece

Yasihiro Yoshiga

Japan

Flavia Del Porto

Italy

Cecilia Hertig

Argentina

Mustafa Cikirikcioglu

Switzerland

Olivier Bouchot

France

Stelios Tzeis

Greece

Fred Buckhold

USA

Andreas Schneider

Germany

S E Kjeldsen

Norway

Sante Pierdomenico

Italy

Iddo Ben-Dov

Israel

D Tsiapras

Greece

George Makavos

Greece

Tain-Yen Hsia

UK

Gemma Sánchez-Espín

Spain

Carlos Velazquez

Spain

Virginia Miller

USA

Ahmet Can

Turkey

Giampaolo Morciano

Italy

Fausto Rigo

Italy 
Suzanne Fredericks

Canada

Jacek Bil

Poland

Demos Katritsis

Greece

Xenophon Costeas

Greece

Sushmita Parashar

USA

Rosa Sicari

Italy

Elizabeth Cross

UK

Sanjay Chatterjee

India

Chirag Bavishi

USA

Tudor Pörner

Germany

Yves St-Pierre

Canada

Manlio Marquez

Mexico

Robert Scragg

New Zealand

Takafumi Okura

Japan

Sumusu Ogawa

Japan

Tomasz Guzik

UK

Senbeta Abdissa

Ethiopia

Zsolt Bagyura

Hungary

Mohamed Abdel Rahman

Egypt

John Saxon

USA

Delphine De Smedt

Belgium

Yumei Gu

Belgium
Johann Auer

Austria

Stylianos Kapiotis

Austria

Jens Jordan

Germany

Dorota Drozdz

Poland

Janne Maier

USA

Catherine Bagot

UK

Stefania Battista

Italy

Roberto Gambino

Italy

Brian Graham

USA

Solomon Abebe

Ethiopia

A.K.M. Islam

Bangladesh

K.M.H.S. Haque

Bangladesh

Michael Toth

USA

Carlo Barbetta

Italy

Alison Short

Australia

Joanne Loewy

USA

Kun-Ta Chou

Taiwan

Fatima Cintra

Brazil

Neil Heron

UK

Rohan Khera

USA

Agnieszka Olszanecka

Poland

Amenah Jaghoori

Australia
Tetsuo Sasano

Japan

Wai-Meng Kwok

USA

Alexandros Briasoulis

USA

Mehmet Birhan Yilmaz

Turkey

Emmy Okello

Uganda

Markus Ferrari

Germany

Jiunn-Lee Lin

Taiwan

T Leiria

Brazil

Gil Salles

Brazil

Andrea Schrepper

Germany

Renzo Levi

Italy

Peter Weeke

Denmark

Christina Werner

Germany

Seong-Kyu Lee

Republic Of Korea

Jeffrey Breall

USA

Oriol Rebagliato Nadal

Spain

Andrew Don-Wauchope

Canada

Panagiota Christia

USA

Antonio Frangieh

Switzerland

Laurence G. Howes

Australia

Ju-Yi Chen

Taiwan

Ales Linhart

Czech Republic 
Mariano Pellicano

Belgium

Themistoklis Maounis

Greece

John Palios

Greece

Liwen Li

China
Emmanouil Kanoupakis

Greece

Anne Child

UK

Cristiana Catena

Italy

Gavin Norton

South Africa
Muharrem Akin

Germany

Celestino Sardu

Italy 